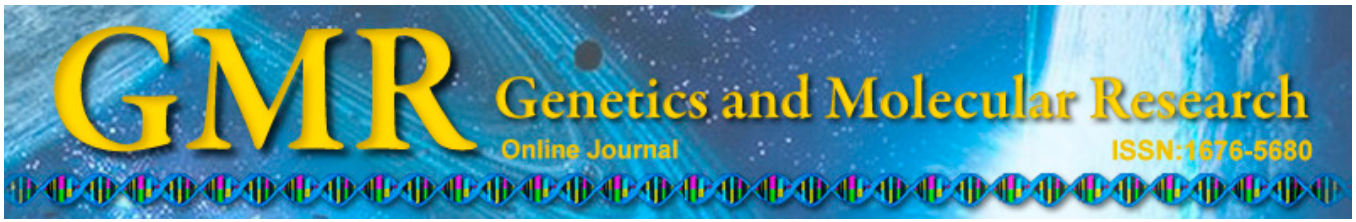

\title{
Clinical value of concurrent radiochemotherapy in cervical cancer and comparison of ultrasonography findings before and after radiochemotherapy
}

\author{
W.M. Yan $^{1 *}$, X.Z. Li ${ }^{2 *}$, Z.L. Yu ${ }^{1}$, J. Zhang ${ }^{1}$ and X.G. Sun ${ }^{1}$ \\ ${ }^{1}$ Department of Radiation Oncology, \\ Affiliated Hospital of Inner Mongolia Medical College, Mongolia, China \\ ${ }^{2}$ Department of Ultrasonic Diagnosis, \\ Affiliated Hospital of Inner Mongolia Medical College, Mongolia, China \\ *These authors contributed equally to this study. \\ Corresponding author: W.M. Yan \\ E-mail: yanwenming9@sohu.com
}

Genet. Mol. Res. 14 (2): 3633-3639 (2015)

Received July 7, 2014

Accepted October 7, 2014

Published April 17, 2015

DOI http://dx.doi.org/10.4238/2015.April.17.13

\begin{abstract}
Herein, we investigated the clinical value of concurrent radiochemotherapy for patients with advanced cervical cancer and its effects on adverse clinical symptoms. Forty patients with cervical cancer were recruited from January 2011 to January 2014 for this study. Participants were randomly allocated into a test or control group, with 20 patients in each group. Patients in the test group were treated with concurrent radiochemotherapy, whereas patients in the control group received only traditional radiotherapy. At the end of the observation period, clinical efficacy in the two groups was compared. Patients were followed up for 2 years, and the rates of recurrence, survival, and complications were compared; ultrasonographic findings before and after radiotherapy were also correlated. Patients in the test group who received concurrent radiochemotherapy showed significantly
\end{abstract}


higher clinical efficacy than the control group at the end of treatment cycles. After 2 years of follow-up, the rates of recurrence, mortality, and complications were all significantly lower in the test group than in the control group $(\mathrm{P}<0.05)$. Comparison of ultrasonographic findings before and after radiochemotherapy showed that the size of the tumor was significantly smaller in patients after concurrent radiochemotherapy. Compared with traditional radiotherapy, concurrent radiochemotherapy significantly improved clinical outcomes in patients with advanced cervical cancer. Concurrent radiochemotherapy also enhanced the rate of survival and decreased the rate of relapse, with enhanced clinical safety and no significant side effects. Thus, concurrent radiochemotherapy can be more broadly applied in the treatment of advanced cervical cancer.

Key words: Cervical cancer; Concurrent radiochemotherapy; Ultrasonography

\section{INTRODUCTION}

Cervical cancer is one of the most common human carcinomas and is prevalent worldwide. It is the most frequent neoplasm among all genital cancers in women and is the most common malignant tumor in women. Its incidence and prevalence show significant regional differences. Cervical cancer in China has unique characteristics in terms of its geographical distribution: areas with a high incidence of cervical cancer are often connected in clusters, and clusters are also present in provincial cities and counties. The incidence in rural areas is higher than in cities, and is also higher in mountainous areas compared to plains (Eifel et al., 2004; Colagrande et al., 2006). The mortality rate of cervical cancer is the fourth highest considering total mortality of all cancers and the second highest among all genital cancers in women. Women in the fourth and fifth decades face the highest risk of cervical cancer, and there is another peak of morbidity in the sixth and seventh decades. Cervical cancer is rare in women in their twenties. Radiotherapy is commonly used to treat cervical cancer, even if it is associated with clinical benefits that are not satisfactory as the rates of recurrence and complications are relatively high. With developments in medical science, radiotherapy combined with concurrent chemotherapy is increasingly used in the treatment of cervical cancer. Concurrent radiochemotherapy refers to the simultaneous application of chemotherapy and radiotherapy and can enhance the effects of radiotherapy by adding low-dose chemotherapy (Vokes and Weichselbaum, 1990). In concurrent radiochemotherapy, patients receive a small dose of daily irradiation in culmination to a substantial dose that is adequate for the treatment of cancer. Radiotherapy is carried out from Monday to Friday. The course of treatment usually lasts for 4-8 weeks, depending on the dose of single irradiation sessions. In order to enhance clinical efficacy, minimize postoperative adverse reactions, and reduce rates of relapse and mortality, our hospital has carried out concurrent radiochemotherapy for patients with cervical cancer from January 2011 to January 2014, and favorable outcomes have been achieved. The procedures used are reported herein.

\section{MATERIAL AND METHODS}

Forty patients with cervical cancer undergoing treatment at our hospital from Janu- 
ary 2011 to January 2014 were recruited for the present study. All patients underwent clinical examinations, had histopathology data available, and had no medical history of cardiac, liver, renal, or other diseases. Clinical symptoms of all patients were compatible with cervical cancer as defined by the International Federation of Gynecology and Obstetrics (FIGO). Among all patients, there were 19 cases with stage IIb, 16 cases with stage III, and 5 cases with stage IV disease. The research was approved by the local Ethics Committee, and all participants and medical personnel signed formal written consent agreement, which ensures that participation in the study was based on voluntary intention. Participants were randomly allocated into two groups: a test group and a control group. In the test group, the age of participants ranged from 29 to 63 years, with a mean age of $48.28 \pm 738$; there were 10 cases with stage IIb, 8 cases with stage III, and 2 cases with stage IV. In the control group, the age of participants ranged from 31 to 62 years, with a mean age of $47.82 \pm 6.89$; there were 9 cases with stage IIb, 8 cases with stage III, and 3 cases with stage IV. There were no significant differences in patient demographics between the two groups of patients $(\mathrm{P}>0.05)$, and thus comparison between the two groups has clinical validity.

\section{Methods}

The control group received traditional radiotherapy, five times a week, with a dose of 2 Gy at each session. Initially, two large field irradiations with a dose of $30 \mathrm{~Gy}$ were delivered to the anterior and posterior walls of the pelvic cavity. When this large field irradiation culminated to a designated dose, it was changed to a four-field irradiation; caution should be taken to place a lead sheet in the middle of the irradiation area to protect the adjacent tissues such as the bladder and rectum. The irradiation dose of the four-field technique was $20 \mathrm{~Gy}$ for consecutive 5 days. If the size of the cervical tumor was relatively large, a vaginal cylinder could be applied twice to remove the tumor according to the conditions of the disease.

The test group received concurrent radiochemotherapy, i.e., concurrent chemotherapy administered on the basis of traditional radiotherapy used in the control group. Concurrent chemotherapy was administered only during the period of external irradiation, with weekly cycles of treatment. One day prior to chemotherapy (cisplatin $40 \mathrm{mg}$, intravenously for 4 consecutively weeks), patients were administered oral dexamethasone as prophylaxis for chemotherapy-induced nausea and vomiting.

\section{Observational parameters}

For short-term clinical efficacy of treatment, National Health Organization (WHO) classification of the Response Evaluation Criteria for solid tumors was used: complete remission (CR), partial remission (PR), stable disease (SD), and progressive disease (PD). CR (Lim et al., 2011) refers to the disappearance of all lesions and no incidences of relapse beyond 4 weeks after the disappearance of lesions. PR refers to at least a 30\% decrease in the sum of the longest diameter of baseline lesions, with no new lesions for 4 consecutive weeks or longer. SD refers to at least a $20 \%$ increase in the sum of the longest diameter of baseline lesions, or the appearance of new lesions. PD refers to less than 30\% decrease in the sum of the longest diameter of baseline lesions, or less than $20 \%$ increase in the sum of the longest diameter of baseline lesions. The final clinical efficacy was calculated as the sum of CR, PR, and SD (Schlecht et al., 2001). 
The rates of adverse reactions in the two groups after the treatment were also compared, and adverse reactions involving bone marrow inhibition, gastrointestinal allergy, cystitis or urethritis were used as observational parameters (Harry, 2008).

Lastly, in terms of the long-term clinical efficacy of cancer treatment, 24-month follow-up was carried out after the treatment cycle ended. The rates of relapse and mortality during follow-up were compared.

\section{Statistical analysis}

The SPSS16.0 package was used for statistical analysis. Staging of cancer, incidence of adverse reactions, and long-term clinical efficacy are reported as percentages. Qualitative data were measured using a chi-square test.

\section{RESULTS}

\section{Short-term clinical efficacy}

After the cycle of treatment was finished, staging of the two groups of patients was clinically examined. The results suggested that patients in the test group who received concurrent radiochemotherapy had significantly better short-term outcomes than did patients in the control group $(\mathrm{P}<0.05$; Table 1$)$.

Table 1. Comparison of the short-term clinical efficacy between groups.
\begin{tabular}{lcccccc}
\hline Group & $\mathrm{N}$ & $\mathrm{CR}(\%)$ & PR $(\%)$ & SD $(\%)$ & Total $(\%)$ & PD \\
\hline Test & 20 & $13(65.0)$ & $4(20.0)$ & $2(10.0)$ & $19(95.0)$ & 1 \\
Control & 20 & $8(40.0)$ & $2(10.0)$ & $2(10.0)$ & $12(60.0)$ & 8 \\
$\chi^{2}$ & 2.50 & 0.78 & 0.00 & 7.03 & 7.03 \\
P & $>0.05$ & $>0.05$ & $>0.05$ & $<0.01$ & $<0.01$ \\
\hline CR = complete remission; PR = partial remission; SD = stable disease; PD = progressive disease.
\end{tabular}

\section{Adverse events in the two groups}

After the cycle of treatment had ended, the incidence of adverse events in the two groups of patients was compared. No significant differences between the group who received concurrent radiochemotherapy and the control group receiving traditional radiotherapy were seen. The results are shown in Table 2.

Table 2. Comparison of adverse reactions between groups.
\begin{tabular}{lccccc} 
Group & $\mathrm{N}$ & Bone marrow inhibition & Gastrointestinal allergy & Cystitis & Urethritis \\
\hline Test & 20 & 3 & 3 & 2 & 1 \\
Control & 20 & 2 & 3 & 1 & 0 \\
$\mathrm{P}$ & & $>0.05$ & $>0.05$ & $>0.05$ & $>0.05$ \\
\hline
\end{tabular}

\section{Comparison of long-term clinical efficacy}

After the cycle of treatment was finished, patients were followed-up for 24 months. Patient conditions were reviewed every 2 weeks. The rates of relapse and mortality in the test group were significantly lower than those in the control group $(\mathrm{P}<0.05)$. The results are detailed in Table 3 . 
Table 3. Comparison of long-term clinical efficacy between groups.

\begin{tabular}{lccc}
\hline Groups & $\mathrm{N}$ & Relapse (\%) & Mortality (\%) \\
\hline Test & 20 & 1 & 0 \\
Control & 20 & 7 & 2 \\
$\chi^{2}$ & & 5.63 & 2.11 \\
$\mathrm{P}$ & & $<0.05$ & $>0.05$ \\
\hline
\end{tabular}

\section{DISCUSSION}

Cervical cancer is a malignant tumor usually occurring in the vaginal portion of the cervix or at the junction of squamous epithelial cells at transitional zones and columnar epithelial cells of the endometrium in the cervical canal. It is the most common cancer in the female genital tract, and is associated with substantial mortality and morbidity. Adjuvant radiotherapy is the main treatment modality for treatment of cervical cancer. However, tumors are difficult to eradicate by radiotherapy and there is significant risk of relapse with notable side effects that depend on the individual reaction to radiotherapy. Accordingly, improvements in the therapeutic effect of radiotherapy in this setting have important clinical significance (Lukka et al., 2002; Thomas et al., 2002; Murakami et al., 2007; Li et al., 2008). In this study, we synchronized radiotherapy and chemotherapy for the treatment of cervical cancer, with significant clinical benefit compared to traditional radiotherapy. As shown in Table 1, considering the short-term clinical efficacy immediately after treatment had ended, $19(95 \%)$ patients showed significant improvement. In contrast, in the control group receiving conventional radiotherapy, only 12 patients $(60 \%)$ showed significant improvement. It can thus be concluded that concurrent radiochemotherapy is significantly better than radiotherapy alone for the treatment of cervical cancer $(\mathrm{P}<0.05)$. In terms of postoperative adverse reactions, as shown in Table 2, in the test group receiving concurrent radiochemotherapy during the treatment period, there were three cases of bone marrow inhibition, three cases of gastrointestinal allergy, two cases of cystitis, and one case of urethritis; in the control group, there were two cases of bone marrow inhibition, three cases of gastrointestinal allergy, one case of cystitis, and no cases of urethritis. There was no significant difference between the two groups, suggesting that there are no significant side effects associated with concurrent radiochemotherapy. Detailed clinical observation showed that the therapy in the test group was well tolerated, as expected. In terms of long-term clinical efficacy, as shown in Table 3, after the cycle of treatment had ended, follow-up was performed for 24 months. During that time, only 1 case of relapse was seen in the test group that required readmission, and no mortality. In contrast, there were 7 cases of relapse and 2 mortalities in the control group receiving conventional radiotherapy. These suggest that the beneficial effects of concurrent radiochemotherapy in the treatment of cervical cancer are more pronounced, and are associated with a lower rate of clinical relapse.

Radiotherapy remains an important local treatment of malignant tumors, with about $70 \%$ of cancer patients requiring radiotherapy during treatment; about $40 \%$ of all cancers can be eradicated with radiotherapy (Taylor et al., 2005; Eifel, 2006; Forrest et al., 2010; Lim et al., 2011; Rose et al., 2011). In the treatment of cervical cancer, radiotherapy can kill residual tumor cells present after surgery, which effectively prevents recurrence and metastasis. In addition, concurrent radiochemotherapy can more effectively kill residual cancer cells at the systemic level and minimize the blood supply to tumor cells (Fujii et al., 2008; 
Kim et al., 2010; Lim et al., 2011). Figures 1 and 2 show a representative case in which tumor shrinkage is seen at 1 week after receiving concurrent radiochemotherapy.
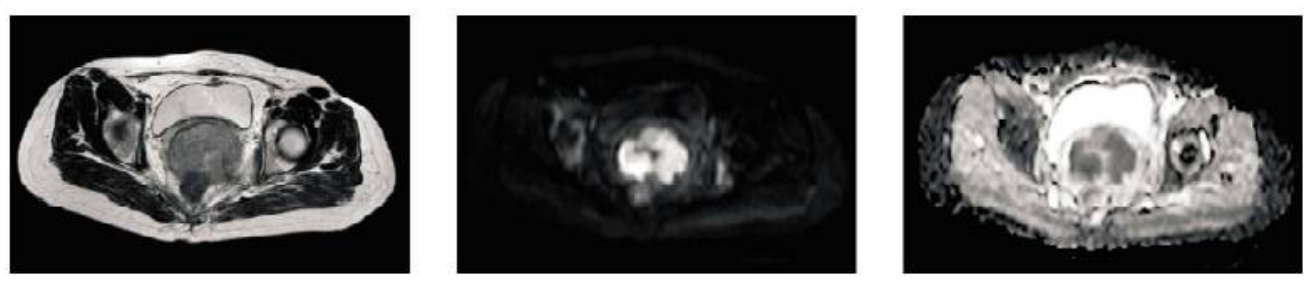

Figure 1. Tumor before concurrent radiochemotherapy.

\section{A}

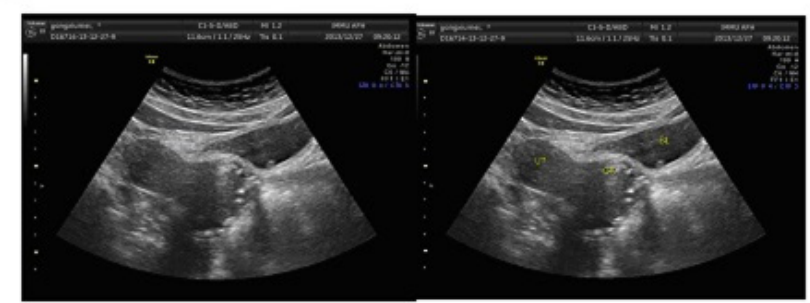

B

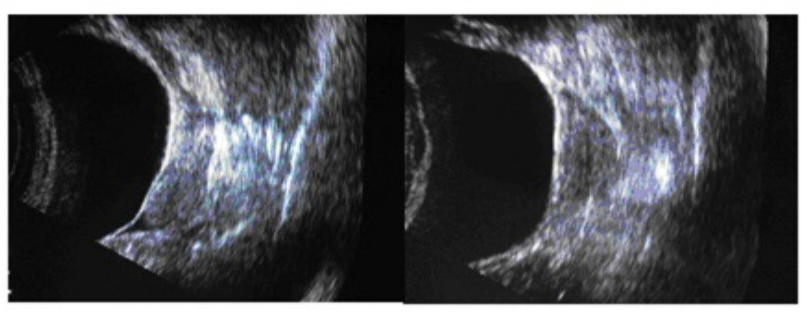

Figure 2. The same tumor before and 1 week after concurrent radiochemotherapy. A. Before concurrent radiochemotherapy. B. After concurrent radiochemotherapy.

It is worth mentioning that applying concurrent chemotherapy to radiotherapy can also provoke harm to patients in terms of adverse reactions such as nausea and dizziness. Thus, it is important to provide supportive care to patients with cervical cancer during concurrent radiochemotherapy to help them overcome potential discomfort. This aspect is also critical in terms of ensuring sustained clinical benefits. Such issues merit further investigation (Kjellberg et al., 2000; Green et al., 2005; Naganawa et al., 2005; Bobek-Billewicz et al., 2010).

\section{REFERENCES}

Bobek-Billewicz B, Stasik-Pres G, Majchrzak H and Zarudzki L (2010). Differentiation between brain tumor recurrence and radiation injury using perfusion, diffusion-weighted imaging and MR spectroscopy. Folia Neuropathol. 48: $81-92$.

Colagrande S, Carbone SF, Carusi LM, Cova M, et al. (2006). Magnetic resonance diffusion-weighted imaging: extraneurological applications. Radiol. Med. 111: 392-419.

Eifel PJ (2006). Chemoradiotherapy in the treatment of cervical cancer. Semin. Radiat. Oncol. 16: 177-185. 
Eifel PJ, Winter K, Morris M, Levenback C, et al. (2004). Pelvic irradiation with concurrent chemotherapy versus pelvic and para-aortic irradiation for high-risk cervical cancer: an update of radiation therapy oncology group trial (RTOG) 90-01. J. Clin. Oncol. 22: 872-880.

Forrest JL, Ackerman I, Barbera L, Barnes EA, et al. (2010). Patient outcome study of concurrent chemoradiation, external beam radiotherapy, and high-dose rate brachytherapy in locally advanced carcinoma of the cervix. Int. J. Gynecol. Cancer 20: 1074-1078.

Fujii S, Matsusue E, Kigawa J, Sato S, et al. (2008). Diagnostic accuracy of the apparent diffusion coefficient in differentiating benign from malignant uterine endometrial cavity lesions: initial results. Eur. Radiol. 18: 384-389.

Green J, Kirwan J, Tierney J, Vale C, et al. (2005). Concomitant chemotherapy and radiation therapy for cancer of the uterine cervix. Cochrane Database Syst. Rev. 3: CD002225.

Harry VN, Semple SI, Gilbert FJ and Parkin DE (2008). Diffusion-weighted magnetic resonance imaging in the early detection of response to chemoradiation in cervical cancer. Gynecol. Oncol. 111: 213-220.

Kim J, Kim MK, Lee JK, Kim JH, et al. (2010). Intakes of vitamin A, C, and E, and beta-carotene are associated with risk of cervical cancer: a case-control study in Korea. Nutr. Cancer 62: 181-189.

Kjellberg L, Hallmans G, Ahren AM, Johansson R, et al. (2000). Smoking, diet, pregnancy and oral contraceptive use as risk factors for cervical intra-epithelial neoplasia in relation to human papillomavirus infection. Br. J. Cancer 82: 1332-1338.

Li S, Xue HD, Wang XH, Sun F, et al. (2008). MR diffusion weighted imaging for evaluation of radiotherapeutic effects on rabbit VX2 tumor model. Chin. Med. Sci. J. 23: 172-177.

Lim K, Small W Jr, Portelance L, Creutzberg C, et al. (2011). Consensus guidelines for delineation of clinical target volume for intensity-modulated pelvic radiotherapy for the definitive treatment of cervix cancer. Int. J. Radiat. Oncol. Biol. Phys. 79: 348-55.

Lukka H, Hirte H, Fyles A, Thomas G, et al. (2002). Concurrent cisplatin-based chemotherapy plus radiotherapy for cervical cancer - a meta-analysis. Clin. Oncol. 14: 203-212.

Murakami R, Sugahara T, Nakamura H, Hirai T, et al. (2007). Malignant supratentorial astrocytoma treated with postoperative radiation therapy: prognostic value of pretreatment quantitative diffusion-weighted MR imaging. Radiology 243: 493-499.

Naganawa S, Sato C, Kumada H, Ishigaki T, et al. (2005). Apparent diffusion coefficient in cervical cancer of the uterus: comparison with the normal uterine cervix. Eur. Radiol. 15: 71-78.

Rose BS, Aydogan B, Liang Y, Yeginer M, et al. (2011). Normal tissue complication probability modeling of acute hematologic toxicity in cervical cancer patients treated with chemoradiotherapy. Int. J. Radiat. Oncol. Biol. Phys. 79: 800-807.

Schlecht NF, Kulaga S, Robitaille J, Ferreira S, et al. (2001). Persistent human papillomavirus infection as a predictor of cervical intraepithelial neoplasia. JAMA 286: 3106-3114.

Taylor A, Rockall AG, Reznek RH and Powell ME (2005). Mapping pelvic lymph nodes: guidelines for delineation in intensity-modulated radiotherapy. Int. J. Radiat. Oncol. Biol. Phys. 63: 1604-1612.

Thomas DB, Ray RM and Qin Q (2002). Risk factors for progression of squamous cell cervical carcinoma in-situ to invasive cervical cancer: results of a multinational study. Cancer Causes Control 13: 683-690.

Vokes EE and Weichselbaum RR (1990). Concomitant chemoradiotherapy: rationale and clinical experience in patients with solid tumors. J. Clin. Oncol. 8: 911-934. 